\title{
Intuitionistic Fuzzy Relational Calculus: an Overview
}

\author{
Chris Cornelis, Glad Deschrijver, Martine De Cock, Etienne Kerre
}

\begin{abstract}
This paper presents an attempt to embed and unify research on intuitionistic fuzzy (IF) relations into a broad, formal yet intuitive framework inspired by fuzzy relational calculus. The need for the resulting "IF relational calculus" is motivated in semantical terms of vagueness and uncertainty; its basic definitions and results are introduced, and opportunities and challenges specific to intuitionistic fuzzy set (IFS) theory are highlighted.
\end{abstract}

Index Terms - Intuitionistic Fuzzy Set Theory, Relational Calculus

\section{INTRODUCTION}

$\mathrm{F}$ UZZY relational calculus originated as a domain of study in the seventies and the early eighties by the seminal works of Zadeh [1] and Bandler and Kohout [2]; later on, this topic was eagerly embraced by the Fuzziness and Uncertainty Modelling Research Unit (FUM in short) at Ghent University, and never ceased to be a prominent feature of its research, both theoretical and oriented to applications such as fuzzy morphology for image processing, the representation of linguistic modifiers, approximate reasoning, fuzzy relational databases, fuzzy preference structures, fuzzy rough sets,... For a comprehensive introduction to fuzzy relational calculus, we refer to [3] and the relevant chapters in [4] and [5].

In this paper, we aim to familiarize the reader with the basic theory of relational calculus, directly applied to intuitionistic fuzzy sets (IFSs). By the identification of the latter as a special kind of L-fuzzy sets (Goguen, [6]), the extension will be fairly straightforward but nonetheless instructive: by compounding our results with a cognitive interpretation, we aim to divulge a new, relational view of IFS theory, which will benefit the further crossfertilization between the theories involved (fuzzy and intuitionistic fuzzy logic in broad sense) and ultimately lead to more sophisticated applications.

The paper is conceived as follows: in section 2, we recall fuzzy sets, L-fuzzy sets and intuitionistic fuzzy sets and consider their connection. Section 3 introduces the concept of an IF relation, its interpretation and its most important associated operations, c.q. : composition, projection, cylindrical extension. In section 4 , as two concrete applications of the standard operations we study the intuitionistic compositional rule of inference (ICRI), and the representation of linguistic modifiers, and motivate their use in the light

Chris Cornelis and Martine De Cock would like to thank the Fund for Scientific Research-Flanders (FWO) for funding the research elaborated on in this paper.

Chris Cornelis, Glad Deschrijver, Martine De Cock and Etienne Kerre are with the Department of Mathematics and Computer Science, Ghent University, Fuzziness and Uncertainty Modelling Research Unit,Krijgslaan 281 (S9), B-9000 Gent, Belgium of IFS theory. Section 5 concludes with some pointers for future work.

We wish to stress that relational calculus is definitely not a novel notion to the IFS community. Notably, P. Burillo and $\mathrm{H}$. Bustince have studied a panoply of properties of IF relations [7], [8] and characterized various structures of IF relations like (pre)orders, transitivity and similarity relations [9]. H. Bustince [10] has further investigated the construction of such structures by means of fuzzy relations. S. De, R. Biswas and A. Roy [11] proposed an application of IF relations in medical diagnosis that was later commented on and given an alternative implementation by E. Szmidt and J. Kacprzyk [12]. G. Deschrijver and E. E. Kerre have generalized the Bandler-Kohout compositions of fuzzy relations and their cuttings [13], [14]; C. Cornelis and G. Deschrijver have outlined an extension of the compositional rule of inference [15]; finally, C. Cornelis and E. Kerre have developed a tentative interpretative scheme for the nascent IF relational calculus in terms of possibility and necessity assignments [16].

\section{Fuzzy Sets, Intuitionistic Fuzzy Sets And L-FUZZY SETS}

Fuzzy sets were introduced by Zadeh in 1965 in [17]. A fuzzy set $A$ in a universe $U$ is a mapping from $U$ to the unit interval $[0,1]$. For any $u \in U$, the number $A(u)$ is called the membership degree of $u$ in $A$. This notion has been extended by Goguen in 1967 to $L$-fuzzy sets [6], where $\left(L, \leq_{L}\right)$ denotes a complete lattice: an $L$-fuzzy set in a universe $U$ is a mapping from $U$ to $L$. Intuitionistic fuzzy sets, defined by Atanassov in 1983 [18], are another generalization of fuzzy sets. While fuzzy sets give a degree of membership of an element in a given set, IFSs give both a degree of membership and of non-membership. Both degrees belong to the interval $[0,1]$, and their sum should not exceed 1. Formally, an IFS $A$ in a universe $U$ was defined as an object of the form $A=\left\{\left(u, \mu_{A}(u), \nu_{A}(u)\right)\right.$ $u \in U\}$, where $\mu_{A}(u)$ is called the "degree of membership of $u$ in $A$ " and $\nu_{A}(u)$, the "degree of non-membership of $u$ in $A^{\prime \prime}$, and where $(\forall u \in U)\left(\mu_{A}(u)+\nu_{A}(u) \leq 1\right) \cdot \pi_{A}(u)=$ $1-\mu_{A}(u)-\nu_{A}(u)$ is called the "hesitation degree of the element $u$ to $A$ ". The class of IFSs in a universe $U$ is denoted $\mathcal{I} \mathcal{F} \mathcal{S}(U)$.

G. Wang and Y. He in [19], and G. Deschrijver and E. E. Kerre in [20] have shown that IFSs are $L$-fuzzy sets w.r.t the complete lattice $\left(L^{*}, \leq_{L^{*}}\right)$ defined by $L^{*}=$ $\left\{\left(a_{1}, a_{2}\right) \in[0,1]^{2} \mid a_{1}+a_{2} \leq 1\right\} ; \quad\left(a_{1}, a_{2}\right) \leq_{L^{*}}\left(b_{1}, b_{2}\right) \Longleftrightarrow$ $a_{1} \leq b_{1} \wedge a_{2} \geq b_{2}$. Hence an IFS $A$ can also be denoted as an $X \rightarrow L^{*}$ mapping. This equivalence gives way to greater flexibility in calculating with membership and non- 
membership degrees, since the pair formed by them is an element of $L^{*}$, and often allows to obtain significantly more compact formulas. The units of $L^{*}$ are given by $0_{L^{*}}=(0,1)$ and $1_{L^{*}}=(1,0)$. In the sequel we will always use the following notation: if $a \in L^{*}$, then $a_{1}$ is the first coordinate and $a_{2}$ is the second coordinate of $a$.

As in fuzzy set theory, the intersection and the union of IFSs are defined by using triangular norms and conorms. [21] An IF t-norm $\mathcal{T}$ is a commutative, associative, increasing $\left(L^{*}\right)^{2}-L^{*}$ mapping which satisfies $\mathcal{T}\left(a, 1_{L^{*}}\right)=a$, for all $a \in L^{*}$. An IF $\mathrm{t}$-conorm $\mathcal{S}$ is a commutative, associative, increasing $\left(L^{*}\right)^{2}-L^{*}$ mapping which satisfies $\mathcal{S}\left(a, 0_{L^{*}}\right)=a$, for all $a \in L^{*}$. The intersection of two IFSs $A$ and $B$, respectively the union, is then defined as, for all $u \in U, A \cap_{\mathcal{T}} B(u)=\mathcal{T}(A(u), B(u)), A \cup_{\mathcal{S}} B(u)=$ $\mathcal{S}(A(u), B(u))$. If we put $\mathcal{T}=(\min , \max )$, i.e. if $\mathcal{T}(a, b)=$ $\left(\min \left(a_{1}, b_{1}\right), \max \left(a_{2}, b_{2}\right)\right)$, for all $a, b \in L^{*}$, then we obtain the definition of intersection given by Atanassov [22]: $A \cap B(u)=\left(\min \left(\mu_{A}(u), \mu_{B}(u)\right), \max \left(\nu_{A}(u), \nu_{B}(u)\right)\right)$. Note that $\mathcal{T}=(\min , \max )$ is the infimum defined w.r.t. the ordering $\leq_{L^{*}}$ on $L^{*}$. Similarly we obtain the traditional definition of the union of IFSs by putting $\mathcal{S}=(\max , \min )$ (this is the supremum w.r.t. the ordering $\leq_{L^{*}}$ defined on $\left.L^{*}\right)$. To define the complement of IFSs, we use an IF negator: a decreasing $L^{*}-L^{*}$ mapping which satisfies $\mathcal{N}\left(0_{L^{*}}\right)=1_{L^{*}}$ and $\mathcal{N}\left(1_{L^{*}}\right)=0_{L^{*}}$. The complement of an IFS is then defined as, for all $u \in U, \operatorname{co} A(u)=\mathcal{N}(A(u))$. If we use the standard negation $\mathcal{N}_{s}$ on $L^{*}$, i.e. $\mathcal{N}_{s}\left(a_{1}, a_{2}\right)=\left(a_{2}, a_{1}\right)$, then we obtain the complement as defined by Atanassov [22]: $\operatorname{co} A(u)=\left(\nu_{A}(u), \mu_{A}(u)\right)$, for all $u \in U$.

\section{Basic Definitions and Operations of Intuitionistic Fuzzy Relational Calculus}

This section is divided into four paragraphs: first we define and position the notion of IF relations with respect to IFS and fuzzy set theory. The next two paragraphs consider the elementary operations on IF relations of composition, projection and cylindrical extension. Finally we list some potential properties of IF relations.

\section{A. Definition and Motivation}

Formally, an IF relation $R$ between (not necessarily all distinct) universes $U_{1}, U_{2}, \ldots, U_{n}(n \geq 1)$ is an IFS in the cartesian product $U_{1} \times \ldots \times U_{n}$. Occasionally, we will use the abbreviation IFR. If $U=U_{1}=\ldots=U_{n}, R$ is called an $n$-ary relation in $U$. Because an IF relation is a special case of an IFS, all operations on IFSs can be applied to IF relations: intersection, union, complement, symmetrical difference, ...

The strength of relations as information models in a knowledge-based system derives from their fundamental ability to describe observed or predicted "connections", expressed as facts or rules, between selected objects of discourse. Crisp relations like $\in, \subseteq,=, \ldots$ have served well in developing rigourous mathematical frameworks. The use of fuzzy relations originated from the observation that reallife objects can be related to each other to a certain degree (just like elements can belong to a fuzzy set to a certain de- gree); in this sense they are able to model vagueness. They are still intolerant of uncertainty, however, since there is no means of attributing reliability or confidence information to the membership degrees. Various frameworks have been conceived to deal with this imperfection, amongst others:

- the certainty factor approach: a crisp real-valued number assigned to a fuzzy relation expresses the confidence in the implied statement [23]

- interval-valued fuzzy (IVF) relations: membership degrees are "softened" by specifying an interval to which the crisp membership degree belongs [24]

- IF relations: membership degrees are "softened" by pairing them with a weakly dependent non-membership degree - second-order fuzzy relations: an extension of the interval-valued approach, where membership degrees themselves become fuzzy sets [1]

We have ranked the alternative approaches according to increasing expressivity: a certainty factor allows only to incorporate general information about the statement as a whole, while the others can differentiate on the level of individual elements of the universe. IF relations, then, are syntactically equivalent with IVF relations: indeed, with every value of $\left(x_{1}, x_{2}\right) \in L^{*}$ corresponds a unique interval $\left[x_{1}, 1-x_{2}\right]$, and vice versa. A thorough study of the implications (cognitive, in terms of interpretations; technical, in terms of available algorithms and tools) of this equivalence will be the object of a future paper. For now, we will concentrate on the IF approach. A possible semantics for IF relations taking inspiration from classical possibility theory was presented in [16]. Basically, the idea is to treat an IF relation as an elastic restriction that allows us to discriminate between the more or less plausible values for a variable. For instance, a statement like "Pete is old" does not allow us to infer Pete's exact age, yet provides some support in favour of the older ages (allowing that those ages are, to a given extent, possible for him), as well as negative evidence against the younger ones (expressing some certainty or necessity that those ages can't in fact be his). We model this observation by indicating how much the original condition "Pete is old" needs to be stretched in order for Pete's age to assume this particular value (this accounts for the designation "elastic"): we assign two separate $[0,1]-$ valued degrees $\mu(u)$ and $\nu(u)$ to every age $u$ in the considered domain, the first one indicating the possibility that Pete's age assumes this particular value and the second one reflecting our certainty that it differs from the given $u$. In classical possibility theory, symmetry between the two indexes is imposed, however, in a sense that from knowledge that it is impossible that Pete is 25 years old $(\mu(25)=0)$, we immediately derive that it is completely certain that he is not $25(\nu(25)=1)$, and more generally from $\mu(u)=\alpha$ follows $\nu(u)=1-\alpha$. Taken together, the various degrees $\mu(u)$ give rise to a fuzzy set. But what if we cannot be sure that the observer is fully credible?

In [16], the authors argued that certainty, as opposed to possibility, is a strong and decisive kind of knowledge, and therefore in gathering evidence, it is sensible to be able to first make tentative conjectures without being pinned down 
on definite commitments, and later fine-tune observations on the basis of estimated confidence in information sources. In other words, we can have varying degrees of trust in an observer, ranging from unconditional inconfidence to full creditworthiness, and we should be able to model that trust accordingly; which can be done conveniently by letting the certainty degree $\nu(u)$ range between 0 and $1-\mu(u)$. This justifies the use of a more general intuitionistic fuzzy, rather than a fuzzy, relation as a model of describing observations.

\section{B. Compositions of IF relations}

A definition for the composition of binary IF relations was first given by Bustince and Burillo [7], [8], in the case that the involved universes are finite. We opted to consider also infinite universes; in this way we can obtain a straightforward extension of the sup- $T$ composition from fuzzy set theory: let $\mathcal{T}$ be an IF t-norm, $R \in \mathcal{I} \mathcal{F S}(U \times V)$ and $S \in \mathcal{I} \mathcal{F S}(V \times W)$. The sup $-\mathcal{T}$ composition of $R$ and $S$ is then the IFR between $U$ and $W$ defined as, for all $(u, w) \in U \times W, R \circ_{\mathcal{T}} S(u, w)=\sup _{v \in V} \mathcal{T}(R(u, v), S(v, w))$. sup denotes the supremum in $L^{*}$. In a similar vein, for an IF t-conorm $\mathcal{S}, R \in \mathcal{I} \mathcal{F} \mathcal{S}(U \times V)$ and $S \in \mathcal{I} \mathcal{F} \mathcal{S}(V \times W)$, the inf $-\mathcal{S}$ composition of $R$ and $S$ is the IFR between $U$ and $W$ defined as, for all $(u, w) \in U \times W, R \circ_{\mathcal{S}} S(u, w)=$ $\inf _{v \in V} \mathcal{S}(R(u, v), S(v, w))$.

The above definition of the sup $-\mathcal{T}$ composition is a generalization of the definition in classical set theory, where the composition of two relations $R \subseteq U \times V$ and $S \subseteq V \times W$ is defined as $R \circ S=\{(u, w) \mid(u, w) \in U \times W \wedge u R \cap S w \neq \varnothing\}$, using the afterset $u R=\{v \mid v \in V \wedge(u, v) \in R\}$ and the foreset $S w=\{v \mid v \in V \wedge(v, w) \in S\}$. This composition is sometimes called the round composition. The relation $R$ can be identified with its characteristic mapping, namely $R(u, v)=1$, if $(u, v) \in R ; R(u, v)=0$ else; and similarly for $S$. It is easily verified that the characteristic mapping of the round composition is then given by $R \circ S(u, w)=\sup _{v \in V} R(u, v) \wedge_{B} S(v, w)$, where $\wedge_{B}$ stands for the Boolean conjunction.

Denote by $R^{t}$ the converse relation of $R$ defined as $R^{t}=$ $\{(v, u) \mid(v, u) \in V \times U \wedge(u, v) \in R\}$. The composition $R \circ S$ is a relation from $U$ to $W$, consisting of those couples $(u, w)$ for which there exists at least one element of $V$ that is in relation $R^{t}$ with $u$ and that is in relation $S$ with $w$. Consider the following example: let $U$ be a set of patients, $V$ a set of symptoms and $W$ a set of illnesses. Define the relation $R \subseteq U \times V$ as $R(u, v)=1$ if patient $u$ shows symptom $v$, and $R(u, v)=0$ if patient $u$ does not show symptom $v$. Define $S \subseteq V \times W$ as $S(v, w)=1$ if symptom $v$ is a symptom of illness $w$, and $S(v, w)=0$ else. Then $R \circ S(u, w)=1$ if patient $u$ shows at least one symptom of illness $w$. Therapists may also want to know whether the symptoms shown by patient $u$ are all symptoms of illness $w$, or whether all the symptoms of illness $w$ are shown by patient $u$, or whether the symptoms shown by patient $u$ are exactly those of illness $w$. Therefore, Bandler and Kohout [2] have defined the triangular compositions. The subcomposition of $R$ and $S$ is defined as $R \triangleleft_{\mathrm{bk}} S=\{(u, w) \mid$ $(u, w) \in U \times W \wedge u R \subseteq S w\}$. The supercomposition of $R$ and $S$ is defined as $R \triangleright_{\mathrm{bk}} S=\{(u, w) \mid(u, w) \in U \times$ $W \wedge S w \subseteq u R\}$. They also defined the ultracomposition of $R$ and $S$ as $R \diamond_{\mathrm{bk}} S=\{(u, w) \mid(u, w) \in U \times W \wedge$ $u R=S w\}$. Then in the example, we have for instance that $R \triangleleft_{\mathrm{bk}} S(u, w)$ is equal to 1 if all symptoms shown by patient $u$ are symptoms of illness $w$ and equal to 0 else.

Bandler and Kohout have shown that the characteristic mappings of these compositions can be found in the following way [2]: $R \triangleleft_{\mathrm{bk}} S(u, w)=\inf _{v \in V} R(u, v) \Rightarrow_{B}$ $S(v, w), R \triangleright_{\mathrm{bk}} S(u, w)=\inf _{v \in V} R(u, v) \Leftarrow_{B} S(v, w)$, and $R \diamond_{\mathrm{bk}} S(u, w)=\inf _{v \in V} R(u, v) \Leftrightarrow_{B} S(v, w)$, where $\Rightarrow_{B}$ and $\Leftrightarrow_{B}$ stand for the Boolean implication and equivalence, and $b \Leftarrow_{B} a$ is defined as $a \Rightarrow_{B} b$.

From the definition of the triangular compositions follows easily that $\operatorname{co}(\operatorname{dom}(R)) \times W \subseteq R \triangleleft_{\mathrm{bk}} S$ and $U \times$ $\operatorname{co}(\operatorname{rng}(S)) \subseteq R \triangleright_{\mathrm{bk}} S$. The first expression means that if $u$ is not in the domain of $R$, then $u$ is in relation $R \triangleleft_{\mathrm{bk}} S$ with all elements of $W$, even if there is no element of $V$ that is in relation $R^{t}$ with $u$. A similar remark holds for the second expression. De Baets and Kerre [25], [26], [27] have therefore introduced the following definitions: the subcomposition $R \triangleleft S=\{(u, w) \mid(u, w) \in U \times W \wedge \varnothing \subset u R \subseteq S w\}$, the supercomposition $R \triangleright S=\{(u, w) \mid(u, w) \in U \times W \wedge \varnothing \subset$ $S w \subseteq u R\}$ and the ultracomposition $R \diamond S=\{(u, w) \mid$ $(u, w) \in U \times W \wedge \varnothing \subset u R=S w\}$.

Before we can give the definition of the triangular compositions of IFRs, we need some preliminary definitions. An IF implicator $\mathcal{I}$ is an $\left(L^{*}\right)^{2}-L^{*}$ mapping which is decreasing in its first component, increasing in its second component and which satisfies the border conditions $\mathcal{I}\left(0_{L^{*}}, 0_{L^{*}}\right)=\mathcal{I}\left(0_{L^{*}}, 1_{L^{*}}\right)=\mathcal{I}\left(1_{L^{*}}, 1_{L^{*}}\right)=1_{L^{*}}$ and $\mathcal{I}\left(1_{L^{*}}, 0_{L^{*}}\right)=0_{L^{*}}$. Clearly this is an extension of both the Boolean implication and the fuzzy implication. An IF implicator $\mathcal{I}$ is called a border implicator if $\mathcal{I}\left(1_{L^{*}}, x\right)=x$, for all $x \in L^{*}$. The afterset $u R$ of $u$ w.r.t. an IFR $R$ between $U$ and $V$ is an IFS in $V$ defined by $u R(v)=R(u, v)$, $\forall v \in V$. The foreset $R v$ of $v$ w.r.t. an IFR $R$ between $U$ and $V$ is the IFS in $U$ defined by $R v(u)=R(u, v)$, $\forall u \in U$. The height of an IFS $A$ in $U, \operatorname{hgt}(A)$ is defined as $\operatorname{hgt}(A)=\sup _{u \in U} A(u)$ (notice that $\operatorname{hgt}(A) \in L^{*}$ ). The plinth of an IFS $A$ in $U, \operatorname{plt}(A)$ is defined as $\operatorname{plt}(A)=\inf _{u \in U} A(u)$. The domain of an IFR $R$ between $U$ and $V$ is the IFS $\operatorname{dom}(R)$ in $U$ defined by $\operatorname{dom}(R)(u)=\operatorname{hgt}(u R)$. The range of an IFR $R$ between $U$ and $V$ is the $\operatorname{IFS} \operatorname{rng}(R)$ in $V$ defined by $\operatorname{rng}(R)(v)=\operatorname{hgt}(R v)$. The converse relation $R^{t}$ of an IF relation $R$ between $U$ and $V$ is the IFR between $V$ and $U$ defined by $R^{t}(v, u)=\left(\mu_{R}(u, v), \nu_{R}(u, v)\right)$, $\forall v \in V, u \in U$.

Let $R$ be an IFR between $U$ and $V, S$ an IFR between $V$ and $W, \mathcal{I}$ an IF implicator, then the triangular sub- and supercomposition of Bandler and Kohout are extended as follows [13]: they are IFRs between $U$ and $W$ defined by, 
for any $u \in U, w \in W$,

$$
\begin{aligned}
& R \triangleleft_{\mathrm{bk}}^{\mathcal{I}} S(u, w)=\inf _{v \in V} \mathcal{I}(R(u, v), S(v, w))=\operatorname{plt} \mathcal{I}(u R, S w), \\
& R \triangleright_{\mathrm{bk}}^{\mathcal{I}} S(u, w)=\inf _{v \in V} \mathcal{I}(S(v, w), R(u, v))=\operatorname{plt} \mathcal{I}(S w, u R),
\end{aligned}
$$

One can verify that in the classical case it holds that $R \triangleleft$ $S=\left(R \triangleleft_{\mathrm{bk}} S\right) \cap(\operatorname{dom}(R) \times \operatorname{rng}(S))$ and $R \triangleright S=\left(R \triangleright_{\mathrm{bk}}\right.$ $S) \cap(\operatorname{dom}(R) \times \operatorname{rng}(S))$. On the other hand we have also $R \triangleleft S=\left(R \triangleleft_{\mathrm{bk}} S\right) \cap(R \circ S)$ and $R \triangleright S=\left(R \triangleright_{\mathrm{bk}} S\right) \cap(R \circ S)$. The first two expressions are generalized to IFRs as [13], for any $u \in U, w \in W$,

$$
\begin{aligned}
R \triangleleft_{\mathrm{b}}^{\mathcal{I}} S(u, w)= & \inf \left(\inf _{v \in V} \mathcal{I}(R(u, v), S(v, w)),\right. \\
& \left.\sup _{v \in V} R(u, v), \sup _{v \in V} S(v, w)\right) \\
= & \inf (\operatorname{plt} \mathcal{I}(u R, S w), \operatorname{hgt}(u R), \operatorname{hgt}(S w)), \\
R \triangleright_{\mathrm{b}}^{\mathcal{I}} S(u, w)= & \inf (\operatorname{plt} \mathcal{I}(S w, u R), \operatorname{hgt}(u R), \operatorname{hgt}(S w)),
\end{aligned}
$$

where inf denotes the infimum in $L^{*}$; while the last two expressions are generalized as [13], for any $u \in U, w \in W$,

$$
\begin{aligned}
& R \triangleleft_{\mathrm{k}}^{\mathcal{T}, \mathcal{I}} S(u, w)= \inf \left(\inf _{v \in V} \mathcal{I}(R(u, v), S(v, w)),\right. \\
&\left.\sup _{v \in V}\left(R(u, v) \wedge_{\mathcal{T}} S(v, w)\right)\right) \\
&= \inf \left(\operatorname{plt} \mathcal{I}(u R, S w), \operatorname{hgt}\left(u R \cap_{\mathcal{T}} S w\right)\right), \\
& R \triangleright_{\mathrm{k}}^{\mathcal{T}, \mathcal{I}} S(u, w)=\inf \left(\operatorname{plt} \mathcal{I}(S w, u R), \operatorname{hgt}\left(u R \cap_{\mathcal{T}} S w\right)\right) .
\end{aligned}
$$

The properties of these compositions are investigated in [13], [14].

\section{Projection and Cylindrical Extension of IF relations}

The ideas of projection and cylindrical extension of fuzzy relations were developed by Zadeh in [1]. Throughout this section, unless stated otherwise, we assume that $R$ denotes an IF relation between $U_{1}, \ldots, U_{n}$, i.e. $R$ is an $U_{1} \times \ldots \times$ $U_{n} \rightarrow L^{*}$ mapping.

Let $I=\left(i_{1}, \ldots, i_{k}\right)$ be a subsequence of $(1,2, \ldots, n)$, $k \leq n$. The complementary sequence of $I$ w.r.t. $(1,2, \ldots, n)$ is $J=\left(j_{1}, \ldots, j_{l}\right)$ such that $\left\{j_{1}, \ldots, j_{l}\right\}=\{1, \ldots, n\} \backslash$ $\left\{i_{1}, \ldots, i_{k}\right\} .{ }^{1} \quad$ The projection of $R$ on $X_{i_{1}} \times \ldots \times$ $X_{i_{k}}$ is denoted $R_{I}$ and defined as $R_{I}\left(u_{i_{1}}, \ldots, u_{i_{k}}\right)=$ $\sup \left\{R\left(x_{1}, \ldots x_{n}\right) \mid\left(x_{j_{1}}, \ldots, x_{j_{l}}\right) \in U_{j_{1}} \times \ldots \times U_{j_{l}}\right.$ and $x_{i_{r}}=$ $\left.u_{i_{r}}, r=1, \ldots, k\right\}, \forall\left(u_{i_{1}}, \ldots u_{i_{k}}\right) \in U_{i_{1}} \times \ldots \times U_{i_{k}}$. As special cases of this definition for $n=2$, we retrieve the domain $(I=(1))$ and the range $(I=(2))$ of a binary IF relation. $R_{I}$ is sometimes called the marginal restriction of $R$ to $U_{i_{1}} \times \ldots \times U_{i_{k}}$. For convenience we will use the notation $R_{i}$ to denote a projection on a single universe $U_{i}$, i.e. $I=(i)$. If it holds that $R\left(u_{1}, \ldots, u_{n}\right)=$ $R_{1} \times \ldots \times R_{n}\left(u_{1}, \ldots, u_{n}\right), \forall\left(u_{1}, \ldots, u_{n}\right) \in U_{1} \times \ldots \times U_{n}$ then $R$ is called separable.

Conversely, starting from an IF relation $R_{I}$ between $U_{i_{1}}, \ldots, U_{i_{k}}$ one can try to find the IF relations between

\footnotetext{
${ }^{1}$ For example, the complementary sequence of $I=(1,7,9)$ w.r.t. $(1,2,3,4,5,6,7,8,9)$ is $J=(2,3,4,5,6,8)$.
}

$U_{1}, U_{2}, \ldots, U_{n}$ such that their projection on $U_{i_{1}} \times \ldots \times U_{i_{k}}$ will equal $R_{I}$. Several IF relations may satisfy that property; the greatest among them, in the sense that all others are subsets of it, will be called the cylindrical extension $\overline{R_{I}}$ of $R_{I}$, an $U_{1} \times \ldots \times U_{n} \rightarrow L^{*}$ mapping given by the following definition: $\overline{R_{I}}\left(u_{1}, \ldots, u_{n}\right)=R\left(u_{i_{1}}, \ldots, u_{i_{k}}\right)$, $\forall\left(u_{1}, \ldots, u_{n}\right) \in U_{1} \times \ldots \times U_{n} . \overline{R_{I}}$ is called cylindrical extension of $R$ because the value of $R$ at each point $\left(u_{1}, \ldots, u_{n}\right)$ equals that at $\left(u_{1}^{\prime}, \ldots, u_{n}^{\prime}\right)$ provided $u_{i_{1}}=u_{i_{1}}^{\prime}, \ldots, u_{i_{k}}=$ $u_{i k}^{\prime}$.

On the other hand, starting from our initial IF relation $R$, the projection $R_{I}$ of $R$ on $U_{i_{1}} \times \ldots \times U_{i_{k}}$ for an arbitrary subsequence $I=\left(i_{1}, \ldots, i_{k}\right), k<n$, of $(1,2, \ldots, n)$ and its associated cylindrical extension $\overline{R_{I}}$ may be constructed. It follows easily that $R \subseteq \overline{R_{I}}$, and hence $R \subseteq \overline{R_{I_{1}}} \cap \overline{R_{I_{2}}} \cap \ldots \cap \overline{R_{I_{s}}}$ where $I_{1}, \ldots, I_{s}$ represent all possible genuine subsequences of $(1,2, \ldots, n)$. Putting $R_{\max }=\overline{R_{I_{1}}} \cap \overline{R_{I_{2}}} \cap \ldots \cap \bar{R}_{I_{s}}$, we find that $R_{\max }$ represents the greatest IF relation between $U_{1}, \ldots, U_{n}$ such that its projections equal that of $R$.

We will motivate the above operations with a simple illustrative example. Suppose that our knowledge about two variables $X$ and $Y$, taking values in the respective universes $U$ and $V$, is summed up by the following statements: " $X$ is $P$ " and " $Y$ is $Q$ ". $P$ and $Q$ are assumed to be IFSs in $U$ and $V$, resp. This implicit conjunction can be considered a restriction " $X$ is $R$ " on the ordered pair $(X, Y)$, where the IF relation $R$ imposes an elastic constraint on the values of the couple $(X, Y)$. Plausibly assuming that $P$ and $Q$ are marginal restrictions of $R$, we propose to model $R$ as the greatest IF relation between $U$ and $V$ (so as not to impose any superfluous conditions) such that $P$ and $Q$ are still its marginals. In other words, $R=\bar{P} \cap \bar{Q}$.

The composition of IF relations has a representation in terms of cylindrical extension: consider IF relations $R$ from $U$ to $V$ and $S$ from $V$ to $W$. For arbitrary IF t-norm $\mathcal{T}$, the sup- $\mathcal{T}$ composition of $R$ and $S$ can be written as $R \circ_{\mathcal{T}} S=$ $\left(\bar{R} \cap_{\mathcal{T}} \bar{S}\right)_{(1,3)}$. In a similar vein, we can express the direct image of an IFS $A$ in $U$ under an IF relation between $U$ and $V$ by using cylindrical extension ${ }^{2}: A_{\circ_{\mathcal{T}}} R=\left(R \cap_{\mathcal{T}} \bar{A}\right)_{2}$.

\section{Useful Properties of binary IF relations}

In this subsection we consider binary IF relations, i.e. IF relations in $\mathcal{I} \mathcal{F} \mathcal{S}(U \times U)$. For an exhaustive treatment of this subject matter we refer to Bustince and Burillo [7], [8], [9], [10].

We say that $R \in \mathcal{I} \mathcal{F} \mathcal{S}(U \times U)$ is reflexive if for every $u \in U, R(u, u)=1_{L^{*}} . R$ is called antireflexive if for every $u \in U, R(u, u)=0_{L^{*}} . R$ is symmetric if for every $(u, v) \in$ $U \times U, R(u, v)=R(v, u) . \quad R$ is called antisymmetrical intuitionistic if for all $(u, v) \in U \times U$, from $u \neq v$ follows $\mu_{R}(u, v) \neq \mu_{R}(v, u), \nu_{R}(u, v) \neq \nu_{R}(v, u)$ and $\pi_{R}(u, v)=$ $\pi_{R}(v, u) . R$ is perfect antisymmetrical intuitionistic if for every $(u, v) \in U \times U$ such that $u \neq v$ and either $\mu_{R}(u, v)>$

\footnotetext{
${ }^{2}$ Note that we are using overloading of the symbol $\circ_{\mathcal{T}}$ for the composition of IF relations.
} 
0 or $\mu_{R}(u, v)=0 \wedge \nu_{R}(u, v)<1$, holds that $\mu_{R}(v, u)=0$ and $\nu_{R}(v, u)=1$. $R$ is called transitive if $R \supseteq L^{*} R \circ_{\mathcal{T}} R$. $R$ is called $C$-transitive if $R \subseteq_{L^{*}} R \circ_{\mathcal{S}} R$.

$R$ is called an IF tolerance relation on $U \times U$ if $R$ is reflexive and symmetric, an IF preorder if it is reflexive and transitive, an IF order if it is an antisymmetrical preorder, an IF similarity relation if it is a symmetrical preorder.

\section{Applications of Intuitionistic Fuzzy Relational Calculus}

\section{A. Intuitionistic Compositional Rule of Inference}

In section III-C, by means of the operations on IF relations, we have laid the foundations of a kind of calculus of flexible restrictions as interpretations of IFR's on the semantical level that allows us to reason with statements coloured by imprecision. Approximate reasoning, then, is a domain of research that attempts to implement this calculus in the solution of everyday problems that cannot be handled adequately by precise techniques because they are either too complex or do not require the precision of an exact, crisp method. Nowadays, people start to accept such "fuzzy systems" as flexible and convenient tools to solve a myriad of ill-defined but otherwise (for humans) straightforward tasks: controlling fluid levels in a reactor, automatical lens focussing in cameras, adjusting an aircraft's navigation to the change of winds, etc. The next step is to try and meet more challenging requirements (e.g. aspects of logical consistency; incorporation of varying facets of imprecision) in order to implement a successful artificial reasoning unit. In terms of trade-off between efficiency and expressiveness, IF relations score very well (compared to fuzzy relations with or without certainty factors on one hand, and second-order fuzzy relations on the other hand), and therefore seem to fit the challenges well. In this section we will study the generalization of the Compositional Rule of Inference, the central tool in approximate reasoning strategies.

We set the scene by providing a simple example. Consider the following statements:

Oskar thinks Kurt is about the same size as him.

Kurt asserts he's a bit smaller than 1 meter.

Moreover, assume Oskar and Kurt are cheeky children with a lively fantasy, and their statements should be taken with a pinch of salt ("a bit smaller" might be an understatement), so that IF relations with $\pi \not \equiv 0$ are in order to model their observations. "a bit smaller than $1 \mathrm{~m}$ " and "about the same size as Kurt" could then be associated to an IFS $A$ and a binary IF relation $R$ in $\mathbb{R}^{+}$respectively (we will not specify them, however). Representing Oskar and Kurt's length by variables $X$ and $Y$ we end up with the following compact expressions:

$$
\begin{aligned}
& X \text { and } Y \text { are } R \\
& X \text { is } A
\end{aligned}
$$

Joining the propositions as we did in the last section, a flexible restriction on the ordered pair $(X, Y)$ emerges:

$$
X \text { and } Y \text { are } R \cap \bar{A}
$$

It should be noted that this statement gathers all information we currently have about $X$ and $Y$. If we want to use this information to make an inference (another restriction) about the value of $Y$, we will do so by taking the marginal restriction of (3) to (the universe of) $Y$. We end up with:

$$
Y \text { is }(R \cap \bar{A})_{2}=R \circ A
$$

An inference about $Y$ thus emerges by composing an IFS with an IF relation. This amounts for the choice of terminology "intuitionistic compositional rule of inference". The composition in general may involve an arbitrary IF t-norm $\mathcal{T}$; we arrive at the following pattern:

$$
\begin{aligned}
& X \text { is } A \\
& (X, Y) \text { is } R \\
& \hline Y \text { is } B=A \circ_{\mathcal{T}} R
\end{aligned}
$$

The example considered a very basic problem, namely that of two interacting variables, where one is characterized in isolation and the approximate relationship between the two is given. In general, any number of relationships between any number of variables may be provided, and an inference about any combination of variables may be inquired. The above procedure can fortunately be easily generalized, as the algorithm below shows (to avoid notational clutter, we describe the steps without referring to actual IF relations): 1. Take the cylindrical extension of all the given IF relations to the cartesian product of the universes of the appearing variables.

2. Take the intersection of these cylindrical extensions. 3. Project the result onto the universe of the variables whose relationship is inquired about.

This scheme was denoted General Rule of Inference. [28] Unlike the case presented in the example, in general the result cannot be written down using IF composition.

The compositional rule of inference and its extension have proven especially useful in rule-based systems. In such an environment, a deduction pattern called Generalized Modus Ponens (GMP):

$$
\begin{array}{ll}
\text { IF } X \text { is } A, \text { THEN } & Y \text { is } B \\
X \text { is } A^{\prime} & Y \text { is } B^{\prime}
\end{array}
$$

can be implemented using the CRI (the rule will be modelled accordingly as an IF relation $R$ ). The study of this pattern for IF relations was conducted by C. Cornelis and G. Deschrijver in [15].

\section{B. Linguistic Modifiers}

A prerequisite for the applicability of the approximate reasoning schemes discussed above is the availability of suitable IFSs for the linguistic statements involved. Constructing them is typically one of the most difficult tasks when developing an application. Therefore it is very useful to have representations of linguistic modifiers such as very and more or less at hand, since they allow for the automatic construction of IFSs for modified terms from IFSs of the original terms. In [29] an extension of Zadehs concentration and dilation operators.$^{2}$ and.$^{\frac{1}{2}}$ was proposed to this 
end. It is however well-known that even in the fuzzy set theoretical case, these operators have the significant shortcoming of keeping the kernel and the support; hence they can not make any distinction between e.g. being old to degree 1 and being very old to degree 1 . In [30] therefore an alternative approach is proposed in which the context is taken into account by means of an IF relation $R$ in $U$ that models approximate equality. In particular, for every IF set $A$ in $U$, more or less $A$ can then be modelled by $A \circ_{\mathcal{T}} R$, the direct image of $A$ under $R$; we will denote it by $R \uparrow_{\mathcal{T}} A$ in this context. Likewise very $A$ can be represented by the superdirect image of $A$ under $R$, defined as

$$
R \downarrow_{\mathcal{I}} A(v)=\inf _{u \in U} \mathcal{I}(R(u, v), A(u))
$$

for all $v$ in $U$. In this scheme $\mathcal{T}$ and $\mathcal{I}$ denote an IF t-norm and an IF implicator as usual. The underlying semantics is that $v$ is very $A$ if all elements resembling $u$ are $A$, and that $u$ is more or less $A$ if an element resembling $u$ is $A$. If $R$ is reflexive (which is a very natural assumption for an IF relation modelling approximate equality) and $\mathcal{I}$ is a border implicator, then this representational scheme respects semantical entailment: very $A \subseteq A \subseteq$ more or less $A$.

The question arises which conditions should be imposed on all "building-bricks" involved, in order for the modification of the IFS $A$ to be some kind of aggregation of modifications of its composing parts. In the representational scheme presented above, this could come down to looking for conditions under which $R \uparrow_{\mathcal{T}} A=\left(\mu_{R} \uparrow_{T} \mu_{A},\left(\operatorname{co} \nu_{R}\right) \downarrow_{I} \nu_{A}\right)$ and likewise $R \downarrow_{\mathcal{I}} A=$ $\left(\mu_{R} \downarrow_{I} \mu_{A},\left(\operatorname{co} \nu_{R}\right) \uparrow_{T} \nu_{A}\right)$, where $\uparrow_{T}$ and $\downarrow_{I}$ denote respectively the fuzzy direct and superdirect image, using a tnorm $T$ on $[0,1]$ and a fuzzy implicator $I$. Furthermore it should also be investigated whether the hesitation degree should or should not be changed through modification of the IFS, and how this can be met mathematically.

\section{Conclusion}

Replying to the need for knowledge-based systems possessing more sophisticated representation and manipulation skills tolerant of vagueness and uncertainty, we introduced Intuitionistic Fuzzy Relational Calculus as a framework for processing imprecise data represented as IF relations. In IF relational calculus, IF relations describe incomplete and/or uncertain information on the values a variable may assume. The added value of IF relations over fuzzy relations lies in their ability to capture varying degrees of reliability or confidence of information. Knowledge manipulation is done by the IF extension of the General Rule of Inference, a special instance of which, the Intuitionistic Compositional Rule of Inference, applies amongst others to relational knowledge expressed as if-then rules, and is directly applicable in IF expert systems.

\section{REFERENCES}

[1] L.A. Zadeh The concept of a linguistic variable and its application to approximate reasoning I, Information sciences, 8, 1975, 199249.

[2] W. Bandler, B. Kohout Fuzzy Relational Products as a Tool for Analysis and Synthesis of the Behaviour of Complex Natural and
Artificial Systems, Theory and Application to Policy Analysis and Information Systems (P. Wang, S. Chang eds.) Plenum Press, New York, 1980, 341-367.

[3] E.E. Kerre, A Walk Through Fuzzy Relations and Their Application to Information Retrieval, Medical Diagnosis and Expert Systems, Analysis and Management of Uncertainty: Theory and Applications (B. Ayyub, M. Gupta, L. Kanal, eds.), North-Holland, Amsterdam, 1993, 141-151.

[4] E.E. Kerre, Introduction to the Basic Principles of Fuzzy Set Theory and Some of its Applications, Communication and Cognition, 1993.

[5] E.E. Kerre, Fuzzy Sets and Approximate Reasoning, XianJiaotong University Press, 1999.

[6] J. Goguen, L-fuzzy Sets, Journal Math. Anal. Appl. 18, 1967, $145-174$

[7] P. Burillo, H. Bustince Intuitionistic Fuzzy Relations (part I), Mathware and Soft Computing 2, 1995, 5-38.

[8] P. Burillo, H. Bustince Intuitionistic Fuzzy Relations (part II), Mathware and Soft Computing 2, 1995, 117-148.

[9] H. Bustince, P. Burillo Structures on Intuitionistic Fuzzy Relations, Fuzzy Sets and Systems 78, 1996, 293-303.

[10] H. Bustince Construction of Intuitionistic Fuzzy Relations with Predetermined Properties, Fuzzy Sets and Systems 109, 2000, $379-403$.

[11] S.K. De, R. Biswas, A.R. Roy Some operations on intuitionistic fuzzy sets Fuzzy Sets and Systems 114 (3), 2000, 477-484.

[12] E. Szmidt, J. Kacprzyk, Intuitionistic Fuzzy Sets in Intelligent Data Analysis for Medical Diagnosis Lecture Notes in Computer Science 2074, (V. Alexandrov, J. Dongarra, B. Juliano, R. Renner, C. Tan, eds.), 2001, 263-271.

[13] G. Deschrijver, E.E. Kerre On the Composition of Intuitionistic Fuzzy Relations, Submitted to Fuzzy Sets and Systems.

[14] G. Deschrijver, E. Kerre On the Cutting of Intuitionistic Fuzzy Compositions, Submitted to Fuzzy Sets and Systems.

[15] C. Cornelis, G. Deschrijver The compositional rule of inference in an intuitionistic fuzzy logic setting Proceedings of the ESSLLI 2001 Student Session, (Kristina Striegnitz, ed.), 2001, 83-94.

[16] C. Cornelis, E.E. Kerre Reliability of Information: Motivation for an Intuitionistic Theory of Possibility Submitted to Fuzzy Sets and Systems.

[17] L.A. Zadeh Fuzzy sets, Information and Control, 8, 1965, 338353.

[18] K. T. Atanassov Intuitionistic fuzzy sets, VII ITKR's Session, Sofia, June 1983 (Deposed in Central Sci. - Techn. Library of Bulg. Acad. of Sci., 1697/84) (in Bulgarian)

[19] G. Wang, Y. He Intuitionistic fuzzy sets and L-fuzzy sets Fuzzy Sets and Systems, 110(2), 2000, 271-274.

[20] G. Deschrijver, E. E. Kerre, On the relationship between some extensions of fuzzy set theory, Accepted to Fuzzy Sets and Systems, 2002.

[21] C. Cornelis, G. Deschrijver, E. E. Kerre Classification of Intuitionistic Fuzzy Implicators: an Algebraic Approach. Proceedings of 6th Joint Conference on Information Sciences (H. J. Caulfield, S. Chen, H. Chen, R. Duro, V. Honavar, E. E. Kerre, M. Lu, M. G. Romay, T. K. Shih, D. Ventura, P. P. Wang, Y. Yang, eds.), 2002, 105-108.

[22] K. T. Atanassov Intuitionistic Fuzzy Sets, Physica-Verlag Heidelberg, New York, 1999.

[23] E.H. Shortliffe, B.G. Buchanan A model of inexact reasoning in medicine Mathematical Biosciences, 23, 1975, 351-379.

[24] I.B.Türksen Interval valued fuzzy sets based on normal forms Fuzzy sets and systems, 20, 1986, 191-210.

[25] B. De Baets, E. E. Kerre, Fuzzy relational compositions, Fuzzy Sets and Systems 60, 1993, 109-120.

[26] B. De Baets, E. E. Kerre, Fuzzy relations and applications Advances in Electronics and Electron Physics 89, 1994, 255-324.

[27] B. De Baets, E. E. Kerre, The cutting of compositions, Fuzzy Sets and Systems bf 62, 1994, 295-309.

[28] D. Ruan, E.E. Kerre On the extension of the compositional rule of inference International Journal of Intelligent Systems, 8, 1993, 807-817.

[29] S.K. De, R. Biswas, A.R. Roy An application of intuitionistic fuzzy sets in medical diagnosis, Fuzzy Sets and Systems 117 (2), 2001, 209-213.

[30] M. De Cock, Z. Žabokrtský, E. E. Kerre Representing Linguistic Hedges by L-Fuzzy Modifiers, Proceedings of CIMCA 2001, 2001, 64-72. 